\title{
Bioanalytical and methodological challenges in the evaluation of fetal Cannabis exposure
}

\author{
Jefferson Pereira Silva*,1 \& Mauricio Yonamine ${ }^{1}$ \\ ${ }^{1}$ Department of Clinical \& Toxicological Analyses, School of Pharmaceutical Sciences, University of São Paulo, Av. Prof. Lineu \\ Prestes 580, 13B, 05508-900 São Paulo, Brazil \\ *Author for correspondence: Tel.: +55 113091 2194; silvajp@usp.br
}

First draft submitted: 28 February 2018; Accepted for publication: 1 March 2018; Published online: 17 May 2018

Keywords: alternative matrices $\bullet$ analytical methods $\bullet$ cannabinoids $\bullet$ drug abuse $\bullet$ fetal exposure $\bullet$ meconium $\bullet$ pregnancy • sample preparation • umbilical cord

Cannabis abuse in pregnancy is considered a risk factor in reproductive toxicology. The impact of cannabinoids in utero exposure is closely related to damages to the fetus [1-3]. However, toxicological data are scarce and the clinical assessment of fetal exposure largely depends on the mother's report, which often omits drug use [4]. The securest way to assess the exposure is to apply analytical methods for drug detection. However, when combining two factors such as 'cannabinoids' and 'uterine environment', the analysis demands a greater analytical expertise and must be managed with meticulous planning.

Pregnancy is a highly sui generis physiological and anatomical condition, in which new compartments of unique biochemical composition are built, forming new patterns of fluids (e.g., amniotic fluid, breast milk) and highly specialized tissues (e.g., placenta and umbilical cord). This brand-new body frame directly influences the toxicokinetics of drugs, which massively interferes with analytical decisions. The success of such evaluations depend on crucial questions taken as the heart of analytical pathway to be explored: Which matrix should be chosen? Should this be selected from the maternal or fetal environment? Which analytes should compose the set of target biomarkers? What is the most suitable sample preparation? What is the meaning of the analytical results? All of these raised questions should be answered with caution by the analyst to guarantee reliability of the laboratory report and to overcome the challenges faced in this kind of task.

\section{Maternal-fetal matrices}

Most of the biological samples used to evaluate drug use in the gestational period are considered nonconventional specimens due to the fact that there are few toxicological studies dedicated to them. Analytical methodologies for medicines and drugs can be found in the literature using neonate hair, amniotic fluid, placenta, vernix caseosa and finger nails [5,6], however, their difficult collection and processing, less availability and the possibility of contamination with other fluids are some of the disadvantages of those specimens, making them less promising.

Therefore, to overcome the lack of data and to build an effective evaluation, the choice of sample should match the following requirements:

- Theoretically, the most appropriate matrices to assess exclusively fetal exposure would be those ones linked only to the infant. In these terms, meconium, urine, blood and nail could ensure that the drug has reached the fetal compartment [7]. However, what actually occurs in pregnancy is a certain indissociability between the maternal and the fetal compartment, leading the rates of incorporation of drugs to be intrinsically dependent on maternal circulation, metabolism and health [8,9], which biases the analysis and interpretations of results.

- The matrix must encompass a wide detection window, reflecting most of the gestational period. Matrices that are formed in the first weeks of gestation and those ones that are capable of accumulating xenobiotics are the specimens with these features.

- The ease of collection of sufficient amount of sample is a primordial preanalytical matter, especially in the case of infant biological samples, for which anatomical access may be difficult and the quantity may be scarce. 
Therefore, the analyst has an arduous task of choosing the most appropriate matrix to evaluate fetal exposure. In fact, the samples that encompass most of the above-mentioned features are meconium and umbilical cord.

\section{Selection of biomarkers of fetal exposure to cannabinoids}

The knowledgement on the metabolism, distribution and excretion of cannabinoids and their derivatives in human samples is crucial to choose a suitable set of biomarkers to be assessed in analytical methods.

Cannabis presents hundreds of phytocannabinoids and metabolites, and their levels are still unknown even in conventional and especially in fetal exposure matrices $[10,11]$. Analytically, the ideal selection method is to identify the largest number of compounds (if there is no biomarker in the available literature pointing to a single substance as gold standard) to evaluate efficiently cannabinoids exposure in utero. In theory, all cannabinoids could be valuable for the global assessment of fetal exposure; however, the choice of the analytes set should be based on its representativeness, considering its abundance and ease of analysis. In summary, these are some key analytes that may be useful for fetal exposure assessment:

- $\Delta^{9}$-Tetrahydrocannabinol (THC), the main active component of the Cannabis plant, has high distribution and transport capacity in the human body due to its physicochemical properties, which allow it to cross biological barriers, such as transplacental.

- Two fundamental metabolites of THC, 11-hydroxy- $\Delta^{9}$-THC, a psychoactive product from the hydroxylation of THC, and 11-nor-9-carboxy-THC, the most prevalent inactive metabolite found in human matrices.

- Cannabinol and cannabidiol are representative targets and can be found in fetal matrices, such as meconium.

- The glucuronide forms of all key analytes [12,13].

\section{Sample preparation \& extraction methods}

The strategies for sample preparation and extraction of cannabinoids in maternal-fetal biological matrices (meconium and umbilical cord) should consider the variability of its chemical composition [14]. If they are singular specimens with lack of information on chemical composition, the sample preparation and extraction come to be an almost blind experimental task. Focusing on cleanup pretreatment for the removal of interferents, according to the physicochemical properties of the chosen biomarkers to enrich them, may be a good solution to achieve satisfactory extraction yields and a reasonable sample preparation.

Nowadays, with the development of more and more sensitive equipment, miniaturization has become an analytical trend, in which experiments must be 'low-volume specimens', with minor sample handling, low cost, environmentally friendly, leading to high yields, ease of use and fast performance [15].

\section{Validation of methods for the evaluation of fetal Cannabis exposure}

Validated confirmatory methods are essential for predicting intrauterine exposure to cannabinoids, offering greater safety for results $[10,16]$. Most of the samples used for this purpose are solid or semisolid specimens and fortification is not totally possible. Additionally, there is no certified reference material available for these matrices or a standardized methodology.

Generally, validation can be schematized according to specific international guidelines for bioanalytical validation and detection of illicit substances $[17,18]$. These guidelines suggest some mandatory parameters. However, validation should be considered critically, including not only obligatory but also fundamental parameters for the evaluation of Cannabis use in pregnancy.

Selectivity (endogenous and exogenous), for example, should be determined to eliminate the possible chemical interference of physiologically produced compounds, or even to verify elements derived from medicinal products used during pregnancy or at the time of childbirth, which may hamper the analysis.

Another intrinsic property of cannabinoids and their metabolites refers to the extensive conjugation with glucuronic acid, presenting variable rates among analyte-to-analyte and different matrices [11], requiring hydrolysis, which can increase the extraction yield and the detection sensitivity of analytes in the free form $[19,20]$, especially when the confirmation is performed by gas chromatography.

Briefly, there are two procedures to cleave the glucuronide group: enzymatic and alkaline hydrolysis. The enzymatic reaction occurs by cleavage of the glucuronide group by the $\beta$-glucuronidase enzyme, while the alkaline hydrolysis cleaves this bond through a chemical reaction in a basic medium [19]. 
The matrix effect is also an important factor, because despite the use of high-performance detectors, the analysis can be strongly affected by chemical complexity of the sample. Thus, it is crucial to establish a sample pretreatment capable of efficiently removing the interfering compounds that could cause ion suppression/enhancement [10].

\section{Interpretation of results}

The determination of cannabinoids and metabolites in maternal-fetal matrices can be used to confirm Cannabis use during pregnancy, but it cannot explain cause-effect relationship of clinical complications, since the mechanisms of fetal damages deriving from Cannabis exposure are not completely elucidated. Moreover, the severity of fetal exposure cannot be surely estimated, since analyte concentrations may vary according to several idiosyncratic factors such as physiological and pathological conditions.

\section{Conclusion \& future perspective}

The role of analytical approaches for the assessment of fetal Cannabis exposure is clearly based on the importance of an accurate determination of the biomarkers in maternal-fetal matrices. The analysis must follow a logical experimental design and respond satisfactorily whether fetal exposure to drugs has occurred or not. Due to the lack of information, the ideal scenario would be to establish toxicokinetic correlations of drug incorporation rates between different mother and baby matrices.

Data on fetal health impact are still a bottleneck, since studies of this magnitude would depend heavily on ethical approval and highly controlled experimental conditions. Possibly, with the technological advances, the application of computational prediction and toxicology in silico can fill this gap of data along with in vitro studies about Cannabis and pregnancy.

Finally, the consumption of Cannabis during pregnancy is a growing social issue that involves women of different ages and social classes all over the world, causing severe health problems to mother and neonate. Therefore, more profound studies regarding these compounds in different human samples must continue to be a relevant research topic.

\section{Financial \& competing interests disclosure}

The authors thank Fundação de Amparo à Pesquisa do Estado de São Paulo (FAPESP grant no. 2015/15700-3) and Coordenação de Pessoal de Nível Superior (CAPES PRÓ-FORENSES AUXPE 3419/2014) for supporting our researches on the fetal Cannabis exposure. The authors have no other relevant affiliations or financial involvement with any organization or entity with a financial interest in or financial conflict with the subject matter or materials discussed in the manuscript apart from those disclosed.

No writing assistance was utilized in the production of this manuscript.

\section{References}

1. Szutorisz H, Hurd YL. Epigenetic effects of Cannabis exposure. Biol. Psychiatry 79(7), 586-594 (2016).

2. Jaques SC, Kingsbury A, Henshcke P et al. Cannabis, the pregnant woman and her child: weeding out the myths. J. Perinatol. 34(6), 417-424 (2014).

3. Richardson KA, Hester AK, McLemore GL. Prenatal Cannabis exposure - the first hit to the endocannabinoid system. Neurotoxicol. Teratol. 58, 5-14 (2016).

4. Miniauskiene D, Jurgaitiene D, Strukcinskiene B. Self-reported Cannabis products and other illicit drugs consumption in older school-age children in Northern Lithuania: a comparison between 2006 and 2012. Medicine 50(3), 162-168 (2014).

5. Gray T, Huestis M. Bioanalytical procedures for monitoring in utero drug exposure. Anal. Bioanal. Chem. 388(7), 1455-1465 (2007).

6. Mali N, Karpe M, Kadam V. A review on biological matrices and analytical methods used for determination of drug of abuse. J. Appl. Pharm. Sci. 1(6), 58-65 (2011).

7. Barr DB, Bishop A, Needham LL. Concentrations of xenobiotic chemicals in the maternal-fetal unit. Reprod. Toxicol. 23(3), 260-266 (2007).

8. Berveiller P, Degrelle SA, Segond N, Cohen H, Evain-Brion D, Gil S. Drug transporter expression during in vitro differentiation of first-trimester and term human villous trophoblasts. Placenta 36(1), 93-96 (2015).

9. Al-Enazy S, Ali S, Albekairi N, El-Tawil M, Rytting E. Placental control of drug delivery. Adv. Drug Deliv. Rev. 116, 63-72 (2017).

10. Battista N, Sergi M, Montesano C, Napoletano S, Compagnone D, Maccarrone M. Analytical approaches for the determination of phytocannabinoids and endocannabinoids in human matrices. Drug Test. Anal. 6(1-2), 7-16 (2014).

11. Aizpurua-Olaizola O, Zarandona I, Ortiz L, Navarro P, Etxebarria N, Usobiaga A. Simultaneous quantification of major cannabinoids and metabolites in human urine and plasma by HPLC-MS/MS and enzyme-alkaline hydrolysis. Drug Test. Anal. 9(4), 626-633 (2016). 
12. Scheidweiler KB, Desrosiers NA, Huestis MA. Simultaneous quantification of free and glucuronidated cannabinoids in human urine by liquid chromatography tandem mass spectrometry. Clin. Chim. Acta 413(23), 1839-1847 (2012).

13. Fabritius M, Staub C, Mangin P, Giroud C. Distribution of free and conjugated cannabinoids in human bile samples. Forensic Sci. Int. 223(1-3), 114-118 (2012).

14. Naritaka N, Suzuki M, Sato $\mathrm{H}$ et al. Profile of bile acids in fetal gallbladder and meconium using liquid chromatography-tandem mass spectrometry. Clin. Chim. Acta 446, 76-81 (2015).

15. Jiménez-Díaz I, Vela-Soria F, Rodríguez-Gómez R, Zafra-Gómez A, Ballesteros O, Navalón A. Analytical methods for the assessment of endocrine disrupting chemical exposure during human fetal and lactation stages: a review. Anal. Chim. Acta 892, 27-48 (2015).

16. Moein MM, El Beqqali A, Abdel-Rehim M. Bioanalytical method development and validation: critical concepts and strategies. J. Chromatogr. B 1043, 3-11 (2017).

17. Guidance for the Validation of Analytical Methodology and Calibration of Equipment Used for Testing of Illicit Drugs in Seized Materials and Biological Specimens. United Nations Office on Drugs and Crime, NY, USA (2009). www.unodc.org/documents/scientific/validation_E.pdf

18. SWGTOX. Scientific Working Group for Forensic Toxicology (SWGTOX) standard practices for method validation in forensic toxicology. J. Anal. Toxicol. 37(7), 452-474 (2013).

19. Gray TR, Barnes AJ, Huestis MA. Effect of hydrolysis on identifying prenatal cannabis exposure. Anal. Bioanal. Chem. 397(6), 2335-2347 (2010)

20. Mantovani C de C, Silva JPE, Forster G, Almeida RM de, Diniz EM de A, Yonamine M. Simultaneous accelerated solvent extraction and hydrolysis of 11-nors- $\Delta$ 9-tetrahydrocannabinol-9-carboxylic acid glucuronide in meconium samples for gas chromatography-mass spectrometry analysis. J. Chromatogr. B 1074-1075, 1-7 (2018). 